\title{
Detección y planificación de contenidos problemáticos: el caso de las preposiciones locativas en el aprendizaje de lenguas afines (italiano-español)
}

\author{
Cristina Rodríguez Faneca \\ Universidad de Córdoba \\ 102rofac@uco.es \\ https://dx.doi.org.10.12795/futhark.2019.il4.I I
}

Fecha de recepción: 9.05 .2018

Fecha de aceptación: 23.06.2019

Resumen: En el presente trabajo se realiza una reflexión sobre la importancia de la planificación docente en la enseñanza de contenidos problemáticos. A partir de la observación de un problema en el aula (producción de errores en la comunicación escrita de aprendices hispanohablantes en relación con las preposiciones locativas italianas) presentamos un corpus de errores con el que reflexionar acerca de posibles herramientas, instrumentos y enfoques que podrían ser de utilidad a la hora de abordar dichos contenidos en el aula. El corpus en cuestión recoge 190 producciones escritas de hispanohablantes que estudian italiano como lengua extranjera entre los cursos 2016-2019.

Palabras clave: análisis de errores; preposiciones locativas; lenguas afines; italiano; español

\section{Detection and planning of conflictive contents: the case of locative prepositions in related languages learning (Italian- Spanish)}

\begin{abstract}
This work aims to reflect on the importance of teaching planification when dealing with conflictive contents. After the observation of a problem during several lessons (error production within written communication of Spanishspeaking apprentices as far as Italian locative prepositions is concerned), a corpus containing such errors was compilated so as to be able to reflect on potential tools, instruments or approaches that could be convenient when addressing such contents in the classroom. The above-named corpus contains 190 written
\end{abstract}


productions of Spanish-speakers who studied Italian as foreign language during the courses 2016-2019.

Keywords: error analysis; locative prepositions; related languages; Italian; Spanish

Sumario: Introducción. I. Marco teórico. I.I. La compilación de corpus ad hoc para el estudio de fenómenos en el aula. I.2. El Análisis de Errores en el marco de la planificación de contenidos problemáticos. I.3. El aprendizaje de italiano como LE para hispanohablantes: preposiciones locativas. 2. Objetivo. 3. Metodología. 4. Resultados. 5. Discusión. Conclusiones.

\section{Introducción}

En el presente trabajo se realiza una reflexión sobre la importancia de la planificación de contenidos problemáticos en la enseñanza y sobre cómo la detección y descripción de fenómenos conflictivos a través del material docente puede ayudar a la posterior resolución de los mismos en el aula.

En concreto, tras detectar un contenido problemático (en nuestro caso, las preposiciones locativas), indagamos acerca de su incidencia y de posibles herramientas para mitigarlo. El fenómeno que estudiamos se da en un contexto que también conlleva una dificultad per se: la enseñanza/aprendizaje del italiano para hablantes de español, es decir, lenguas que son afines entre ellas. Por ello, a las dificultades inherentes al aprendizaje de una lengua extranjera (LE) hay que añadir las dificultades propias de las lenguas afines y del argumento que se debe tratar en sí, las preposiciones.

Para llevar a cabo nuestra reflexión partimos de la observación de un fenómeno reiterado en el aula: la gran proporción de errores que presentan las producciones orales y escritas de los aprendices hispanohablantes de italiano en relación con las preposiciones locativas y en comparación con otros posibles errores. Posteriormente aplicamos la metodología de Análisis de Errores (AE) propuesta por Corder (1967) y reflexionamos posteriormente acerca de posibles herramientas, instrumentos y enfoques que podrían ser de utilidad a la hora de abordar dichos contenidos en el aula.

\section{Marco teórico}

I.I. La compilación de corpus ad hoc para el estudio de fenómenos en el aula

La creación de un corpus es el punto de partida para muchos tipos de estudio de corte lingüístico. Su utilidad es amplia, como lo son los análisis que se basan en ellos, aunque en todos prima la necesidad de estudiar la lengua en uso, ya 
que su compilación no suele ser considerada una meta per se. En la definición de corpus propuesta por Sinclair (2004:19) observamos varios puntos que se deben tener en cuenta:

A corpus is a collection of pieces of language text in electronic form, selected according to external criteria to represent, as far as possible, a language or language variety as a source data for linguistic research

En primer lugar, se hace imprescindible contar con un soporte electrónico, sin el cual la utilidad del corpus quedaría limitada. Por otro lado, es importante fijar un criterio de selección de muestras que asegure la representatividad del corpus (Piñol, 2012), de manera que la investigación que lo tome como fuente pueda llevarse a cabo con éxito. Es importante tener en cuenta que un corpus, por su naturaleza, nunca puede abarcar la totalidad de una lengua puesto que no es posible exponer de manera textual todos los ámbitos, situaciones, niveles de especialización ni entes implicados en el proceso comunicativo. Este conflicto se trata de solucionar logrando que el corpus sea representativo, es decir, consiguiendo que las muestras del corpus puedan describir de manera genérica, clara y precisa la interacción que intentemos mostrar.

Por otro lado, Corpas-Pastor, y Seghiri-Domínguez (2006) se ocupan de un tema recurrente en la lingüística de corpus: el número de muestras textuales a partir del cual se garantiza la cientificidad del corpus. Las posiciones en torno a este interrogante son múltiples, aunque para la mayoría de autores un número elevado de textos no es condición sine qua non para la compilación de un corpus, mientras que otros estudiosos defienden que es necesario que la envergadura del corpus sea de, incluso, millones de palabras.

Más allá de proponer un volumen determinado de palabras en un corpus, es indispensable prestar atención a dos parámetros: qué interacción quiere mostrarse y cuál es el propósito de la compilación del corpus (si existe alguno más allá del de mostrar la propia interacción). En el trabajo que nos ocupa, donde pretendemos mostrar un tipo de error muy concreto centrándonos en un grupo reducido de alumnos (nuestro propio grupo) lo interesante es contar con un corpus de pequeño tamaño, cuya compilación es más rápida y práctica y la visualización de datos para obtener resultados es mucho más sencilla.

\section{I.2. El Análisis de Errores en el marco de la planificación de contenidos problemáticos}

No es poco frecuente que el docente se halle reiteradamente ante una situación en la que el alumno comete el mismo error sistemáticamente. En esta situación es necesario que dicho argumento se catalogue como problemático. Así, con la atención puesta en este, se procederá a analizarlo minuciosamente para poder recabar datos suficientes que le otorguen herramientas y recursos para afrontarlo en el aula. 
En nuestro trabajo nos apoyamos, con leves divergencias, en las fases de la metodología de Análisis de Errores (AE) propuesta por Corder (1967), cuyas etapas sugeridas son las de recopilación del corpus, identificación de errores, catalogación de errores, descripción de errores y explicación de los mismos y, por último, propuestas para solventarlos («terapias», en la nomenclatura propuesta por este autor). Esta última etapa solo es de aplicación si el $A E$ tiene un objetivo pedagógico (Quiñones, 2009), en contraposición al AE psicolingüístico, cuyo objetivo es el de obtener información acerca de los procesos de adquisición de lenguas.

Existen ciertas consideraciones que se deben tener en cuenta en cada una de las fases. Dentro de la fase de recopilación del corpus (fase I) se establecen las características de los sujetos participantes en el estudio: lengua materna y origen, extensión de la muestra, habilidad lingüística que se somete a análisis y extensión del mismo, además de su periodicidad. En la fase de identificación de errores (fase 2) debemos determinar qué es un error para su posterior catalogación (fase 3). Una de las clasificaciones de errores más recurrentes dentro de la literatura es la de Vázquez (1992), quien propone 6 criterios taxonómicos: lingüístico, etiológico, comunicativo, pedagógico, pragmático y cultural.

Las fases de descripción y explicación de los errores (fases 4 y 5 ) suelen confundirse entre sí (Quiñones, 2009), por lo que existen multitud de estudios donde ambas fases se tratan indistintamente. Según esta autora, la fase descriptiva tiene que limitarse a responder a la pregunta de qué es lo que está incorrecto, mientras que la fase explicativa tiene que limitarse a responder al interrogante de por qué se ha transgredido una determinada norma lingüistica.

Por último, como hemos mencionado con anterioridad, la propuesta de «terapias» (fase 6) solo es de aplicación si el $A E$ tiene como objetivo la investigación de la interlingua del estudiante.

\section{I.3 El aprendizaje de italiano como LE para hispanohablantes: preposiciones locativas}

Cuando hacemos referencia al aprendizaje de italiano por parte de hispanohablantes hemos de atender a un elemento de complejidad que no aparece siempre que tratamos la enseñanza/aprendizaje de LE: la afinidad entre lenguas.

El hecho de que ambas lenguas sean afines entre ellas trae consigo un gran número de implicaciones e idiosincrasias. Una de las principales es el parentesco léxico entre ambos códigos, que permite un relativo entendimiento entre hispanohablantes e italófonos, ya sea en un plano escrito o hablado (Tomaselli 2008: 6). Esta similitud es puramente superficial: a pesar de que pueden distinguirse sin dificultad todas las categorías gramaticales (artículos, verbos, sustantivos, adverbios y preposiciones), existen multitud de elementos divergentes entre ambas 
lenguas, como el alfabeto, la grafía, la fonética, el acento y el ritmo, por nombrar algunos.

Por ello, en la primera fase de aprendizaje, el alumno posee mayor facilidad para producir discursos haciendo uso de su «interlengua». Este concepto, acuñado por Selinker $(1969,1972)$, hace referencia a una lengua propia de cada estudiante, compuesta por elementos de la lengua materna y de la extranjera y que evoluciona a la par que su aprendizaje. Esta dicotomía (entre diferencias y similitudes, dificultades $y$ facilidades) hace que las estrategias de enseñanza tengan que diferir sustancialmente de las que se esperarían en la didáctica de dos lenguas que no tuviesen una relación de parentesco. A su vez, los factores anteriormente nombrados son precisamente los culpables de que el alumno pueda errar.

Uno de los errores más comunes que comete el estudiante hispanohablante durante el aprendizaje de la lengua italiana son los relativos a las preposiciones. La preposición es un elemento invariable que es regido por la estructura de la oración $y$ es regente del complemento que le sigue, $y$ las preposiciones locativas son aquellas que hacen referencia al lugar como dirección o como lugar en sí (Caballero-Rubio y Hernández-Corral, 1998). El motivo de error se encuentra en la falta de paralelismo entre las preposiciones de ambas lenguas: por ejemplo, el uso de la preposición «a» en español puede equivaler al uso de las preposiciones italianas «in» o «a» (entre otros casos).

En este contexto, la lingüística contrastiva nos dota de herramientas para que, mediante el conocimiento de lo que es igual y diferente en estas dos lenguas, la enseñanza se planifique y dosifique (Caballero-Rubio y Hernández-Corral, 1998) con fundamentos didácticos.

\section{Objetivo}

El principal objetivo de este trabajo es el de ofrecer, tras la detección y descripción de un fenómeno problemático en el aula, posibles herramientas y enfoques que puedan ser de utilidad a la hora de planificar la enseñanza de dicho contenido.

En este caso, el fenómeno analizado es la producción de errores en el uso de preposiciones locativas por parte de aprendices hispanohablantes de italiano como lengua extranjera.

\section{Metodología}

El Análisis de Errores se ha llevado a término siguiendo la metodología propuesta por Corder (1967), centrando el interés en las primeras fases (recopilación del corpus, identificación de errores y catalogación de los mismos 
según criterios lingüísticos) y, sobre todo, en la última fase (propuesta de «terapias»).

Para la compilación del corpus se tomaron producciones escritas extraídas de 190 exámenes de la asignatura Lengua extranjera: italiano del Grado en Turismo de la Universidad de Córdoba durante cuatro cursos académicos consecutivos (entre 2016-2019). Se trata de una asignatura optativa de 6 créditos ECTS cuyo objetivo es que el alumnado alcance un nivel AI-A2 de lengua italiana, por lo que se parte de cero en su aprendizaje.

Las producciones se tomaron tal y como se indica a continuación (tabla I):

\section{Asignatura}

Lengua extranjera: italiano

Lengua extranjera: italiano

Lengua extranjera: italiano

Lengua extranjera: italiano

\section{Titulación}

Grado en Turismo

Grado en Turismo

Grado en Turismo

Grado en Turismo
Fecha

Marzo de 2016

Marzo de 2017

Marzo de 2018

Marzo de 2019
N. ${ }^{\circ}$ de pruebas 50

40

51

49

Tabla I. Producciones contenidas en el corpus. Fuente: elaboración propia.

Una de las consideraciones más importantes durante la etapa de compilación del corpus es que todos los hablantes comparten la misma lengua materna y, si es posible, la misma variedad lingüística (en nuestro caso se trata de aprendices hispanohablantes de italiano que poseen un nivel de Al-A2); sin embargo el tamaño de la muestra es irrelevante puesto que el análisis está centrado en dicho grupo, independientemente de su extensión. Asimismo, se trata de un estudio sincrónico en el que se estudian las producciones escritas en el mismo punto temporal de su formación.

Concretamente, dentro de cada examen, se extrajeron y analizaron las respuestas de un ejercicio en el que se pedía a los alumnos que completasen los huecos de cada oración con una preposición simple y locativa (es decir, se trataba de una pregunta abierta y sin opciones entre las que el alumno pudiese elegir). En este caso, evidentemente, se eliminaron todas las frases cuya respuesta no era una preposición locativa. En definitiva, la prueba rezaba lo siguiente (tabla 2): 


Enunciado
Oración
1
2
3
4

Completa con preposiciones simples (locativas) Contenido

Vivo ( ) Australia, ma sono italiano

Vivo ( ) Spagna, ( ) Cordoba

Oggi ceniamo ( ) Mario

II prossimo volo parte ( ) Pisa
Resp. correcta

in

in, a

da

da/per

Tabla 2. Contenido de la prueba analizada. Fuente: elaboración propia.

Como puede observarse, en algunos casos es posible que más de una preposición, ya sea locativa o no, sea válida. Sin embargo, teniendo en cuenta que lo que se pretende es analizar el uso de estas preposiciones, las variantes válidas para cada oración son las siguientes:

I. Oraciones I y 2: Cualquier preposición incorrecta implicará error.

2. Oración 3: Cualquier preposición incorrecta implicará error, incluso «Oggi ceniamo con Mario», puesto que no se da como respuesta una preposición locativa.

3. Oración 4: Cualquier preposición incorrecta implicará error. No obstante, se consideran correctas dos posibles variantes: «ll prossimo volo parte da/per Pisa».

Al presentar los resultados hemos consignado tres datos fundamentales: (a) el número de veces que se usa cada preposición, (b) el porcentaje de uso una de ellas y (c) el porcentaje total de aciertos y fallos.

En el primer caso, se cuantifica numéricamente el número de veces que cada preposición ha sido utilizada en la respuesta del ejercicio. Aquí también se cuantifica indirectamente el número de usos preposicionales; es decir, cuántas preposiciones distintas han sido objeto de elección por parte de los alumnos.

El porcentaje de uso de cada preposición nos indica, de un modo más ilustrativo, cuáles han sido empleadas más recurrentemente, mientras que el porcentaje total de aciertos, respuestas incorrectas y respuestas en blanco nos proporciona una visión de conjunto más clara en relación a todo el ejercicio y al equilibrio o desequilibrio entre los mismos.

\section{Resultados}

En el caso de la oración I ( $\mathrm{OI}=$ "Vivo in Australia, ma sono italiano») se pretendía que el alumno demostrase conocer el uso de la preposición «in» como complemento circunstancial de lugar que, en italiano, se usa junto con el nombre de un país. 
El porcentaje de acierto en esta oración es muy alto. Con lo cual se observa que, a priori, no existen problemas con esta unidad en relación al resto de preposiciones locativas (tabla 3 ):

\begin{tabular}{|c|c|c|c|c|c|c|c|c|}
\hline \multicolumn{9}{|c|}{ ORACIÓN I } \\
\hline Prep/veces & 2016 & $\%$ & 2017 & $\%$ & 2018 & $\%$ & 2019 & $\%$ \\
\hline in & 44 & $88 \%$ & 34 & $85 \%$ & 40 & $78,40 \%$ & 38 & $77,55 \%$ \\
\hline En blanco & 5 & $10 \%$ & 4 & $10 \%$ & 4 & $7,85 \%$ & 7 & $13,70 \%$ \\
\hline nell' & I & $2 \%$ & 0 & - & 4 & $7,85 \%$ & 2 & $4,10 \%$ \\
\hline $\mathrm{a}$ & 0 & - & I & $2,50 \%$ & 2 & $3,90 \%$ & 1 & $2 \%$ \\
\hline en & 0 & - & I & $2,50 \%$ & I & $1,95 \%$ & 1 & $2 \%$ \\
\hline \multicolumn{9}{|c|}{ Total } \\
\hline Correctas & \multirow{2}{*}{\multicolumn{2}{|c|}{$\begin{array}{l}88 \% \\
12 \%\end{array}$}} & \multirow{2}{*}{\multicolumn{2}{|c|}{$\begin{array}{l}85 \% \\
15 \%\end{array}$}} & \multirow{2}{*}{\multicolumn{2}{|c|}{$\begin{array}{l}78,43 \% \\
21,57 \%\end{array}$}} & \multicolumn{2}{|c|}{$77,55 \%$} \\
\hline $\begin{array}{l}\text { Inc./en } \\
\text { blanco }\end{array}$ & & & & & & & & $45 \%$ \\
\hline
\end{tabular}

En la oración 2 (O2 = «Vivo in Spagna, a Cordoba») se pretendía hacer notar la diferencia entre el uso preposicional para un país y una ciudad. En el primer caso (O2-A), idéntico al anterior, encontramos un porcentaje de acierto igual de alto (tabla 4):

\begin{tabular}{|c|c|c|c|c|c|c|c|c|}
\hline \multicolumn{9}{|c|}{ ORACIÓN 2-A } \\
\hline Prep/veces & 2016 & $\%$ & 2017 & $\%$ & 2018 & $\%$ & 2019 & $\%$ \\
\hline in & 43 & $86 \%$ & 34 & $85 \%$ & $4 I$ & $80,40 \%$ & 42 & $85,70 \%$ \\
\hline En blanco & 5 & $10 \%$ & 4 & $7,85 \%$ & 3 & $5,90 \%$ & 5 & $9,80 \%$ \\
\hline $\mathrm{a}$ & I & $2 \%$ & 2 & $5 \%$ & 2 & $3,90 \%$ & 2 & $4,10 \%$ \\
\hline nell' & I & $2 \%$ & 0 & - & 5 & $9,80 \%$ & 0 & - \\
\hline \multicolumn{9}{|c|}{ Total } \\
\hline Correctas & \multirow{2}{*}{\multicolumn{2}{|c|}{$\begin{array}{l}86 \% \\
14 \%\end{array}$}} & \multirow{2}{*}{\multicolumn{2}{|c|}{$\begin{array}{l}85 \% \\
15 \%\end{array}$}} & \multirow{2}{*}{\multicolumn{2}{|c|}{$\begin{array}{l}80,40 \% \\
19,60 \%\end{array}$}} & \multirow{2}{*}{\multicolumn{2}{|c|}{$\begin{array}{l}85,70 \% \\
14,30 \%\end{array}$}} \\
\hline $\begin{array}{l}\text { Inc./en } \\
\text { blanco }\end{array}$ & & & & & & & & \\
\hline
\end{tabular}

Como hacíamos patente en el caso anterior, en este ejercicio (O2-B) se le pide al alumno que demuestre que conoce la diferencia que existe entre ambos usos preposicionales como complemento circunstancial de lugar. En la primera preposición encontramos un número de aciertos bastante alto, si bien en esta segunda empiezan a multiplicarse los errores.

Es especialmente significativo el hecho de que el número de usos preposicionales totales es superior en este ejemplo (llegando a proponer como soluciones hasta 7 preposiciones incorrectas), lo que pone de manifiesto que las dudas del alumnado se multiplican en este caso (tabla 5): 


\begin{tabular}{|c|c|c|c|c|c|c|c|c|}
\hline \multicolumn{9}{|c|}{ ORACIÓN 2-B } \\
\hline Prep/veces & 2016 & $\%$ & 2017 & $\%$ & 2018 & $\%$ & 2019 & $\%$ \\
\hline a & 32 & $64 \%$ & 29 & $72,50 \%$ & 32 & $62,75 \%$ & 35 & $87,50 \%$ \\
\hline in & 9 & $18 \%$ & 4 & $10 \%$ & 7 & $13,70 \%$ & 3 & $5,90 \%$ \\
\hline En blanco & 6 & $12 \%$ & 3 & $7,50 \%$ & 4 & $7,85 \%$ & 4 & $10 \%$ \\
\hline à & I & $2 \%$ & 0 & - & 0 & - & I & $2,50 \%$ \\
\hline $\mathrm{da}$ & 1 & $2 \%$ & I & $2,50 \%$ & 5 & $9,80 \%$ & 0 & - \\
\hline de & 1 & $2 \%$ & 0 & - & 2 & $3,90 \%$ & 2 & $5 \%$ \\
\hline $\mathrm{di}$ & 0 & - & 1 & $2,50 \%$ & 1 & $1,90 \%$ & 2 & $5 \%$ \\
\hline en & 0 & - & I & $2,50 \%$ & 0 & - & 2 & $5 \%$ \\
\hline 0 & 0 & - & I & $2,50 \%$ & 0 & - & 0 & - \\
\hline \multicolumn{9}{|c|}{ Total } \\
\hline Correctas & \multicolumn{2}{|c|}{$64 \%$} & \multicolumn{2}{|c|}{$72,50 \%$} & \multicolumn{2}{|c|}{$62,75 \%$} & \multicolumn{2}{|c|}{$87,50 \%$} \\
\hline $\begin{array}{l}\text { Inc./en } \\
\text { blanco }\end{array}$ & \multicolumn{2}{|c|}{$36 \%$} & \multicolumn{2}{|c|}{$27,50 \%$} & \multicolumn{2}{|c|}{$37,25 \%$} & \multicolumn{2}{|c|}{$12,50 \%$} \\
\hline
\end{tabular}

En el siguiente caso, la oración 3 (O3, «Oggi ceniamo da Mario»), se observa un número de errores mucho más alto. En esta oración se pretendía, como en el resto, que el alumno usase una preposición que indicase lugar. La preposición «con» no se considera correcta en este caso, y este es uno de los motivos por los que el número de errores es tan alto en esta oración: el alumno ha preferido usar una preposición más familiar con los mismos valores que se le otorgan en castellano (tabla 6):

\begin{tabular}{|c|c|c|c|c|c|c|c|c|}
\hline \multicolumn{9}{|c|}{ ORACIÓN 3} \\
\hline Prep/veces & 2016 & $\%$ & 2017 & $\%$ & 2018 & $\%$ & 2019 & $\%$ \\
\hline da & 19 & $38 \%$ & 15 & $37,50 \%$ & 17 & $33,30 \%$ & 22 & $44,90 \%$ \\
\hline con & 18 & $36 \%$ & 15 & $37,50 \%$ & 21 & $41,20 \%$ & 18 & $36,70 \%$ \\
\hline En blanco & II & $22 \%$ & 9 & $22,50 \%$ & 9 & $17,65 \%$ & 7 & $14,30 \%$ \\
\hline di & 1 & $2 \%$ & 0 & - & 2 & $3,90 \%$ & 2 & $4,10 \%$ \\
\hline$a$ & I & $2 \%$ & I & $2,50 \%$ & 2 & $3,90 \%$ & 0 & - \\
\hline \multicolumn{9}{|c|}{ Total } \\
\hline Correctas & \multirow{2}{*}{\multicolumn{2}{|c|}{$\begin{array}{l}38 \% \\
62 \%\end{array}$}} & \multirow{2}{*}{\multicolumn{2}{|c|}{$\begin{array}{l}37,50 \% \\
62,50 \%\end{array}$}} & \multirow{2}{*}{\multicolumn{2}{|c|}{$\begin{array}{l}33,30 \% \\
66,70 \%\end{array}$}} & \multirow{2}{*}{\multicolumn{2}{|c|}{$\begin{array}{l}44,90 \% \\
55,10 \%\end{array}$}} \\
\hline $\begin{array}{l}\text { Inc./en } \\
\text { blanco }\end{array}$ & & & & & & & & \\
\hline
\end{tabular}

Tabla 6. Preposiciones usadas, aciertos y errores al contestar a la 03.

En virtud de lo que afirma Solsona-Martínez (2006) la preposición «da» registra, dentro del paradigma de las ocho preposiciones propias italianas, un porcentaje de error muy elevado en las producciones de aprendices hispanohablantes de italiano como LE. En el caso que nos ocupa, ello puede deberse a que este uso concreto como preposición locativa no ha sido 
internalizado aún por los alumnos, dado que se encuentran todavía en una fase temprana de aprendizaje.

En la oración 4 (O4, «ll prossimo volo parte da/per Pisa») el número de errores especialmente alto. Un porcentaje muy elevado de alumnos ha optado por usar la preposición «a», otorgándole los mismos valores que la preposición «a» en español debido a la interferencia lingüística y al desconocimiento de la solución correcta, que le lleva a buscar solución en estructuras de su lengua materna.

Un porcentaje muy bajo de alumnos ha decidido usar la preposición «da», ya que han podido identificar correctamente su función como complemento de origen. Lo mismo ocurre con la preposición «per», que también era válida. Normalmente, el alumno tiende a relacionarla con su valor de interés o finalidad o causal, en vez de como complemento circunstancial de lugar (tabla 7):

\begin{tabular}{|c|c|c|c|c|c|c|c|c|}
\hline \multicolumn{9}{|c|}{ ORACIÓN 4} \\
\hline Prep/veces & 2016 & $\%$ & 2017 & $\%$ & 2018 & $\%$ & 2019 & $\%$ \\
\hline a/à & 23 & $46 \%$ & 12 & $30 \%$ & 10 & $19,60 \%$ & 5 & $12,50 \%$ \\
\hline $\mathrm{da}$ & 8 & $16 \%$ & 2 & $5 \%$ & 3 & $5,90 \%$ & 2 & $3,90 \%$ \\
\hline En blanco & 8 & $16 \%$ & 3 & $7,50 \%$ & 2 & $3,90 \%$ & 10 & $25 \%$ \\
\hline $\mathrm{di}$ & 5 & $10 \%$ & I & $2,50 \%$ & 6 & II,70\% & 7 & $17,50 \%$ \\
\hline per & 4 & $8 \%$ & 17 & $42,50 \%$ & 22 & $43,15 \%$ & 19 & $47,50 \%$ \\
\hline in & I & $2 \%$ & 2 & $5 \%$ & 3 & $5,90 \%$ & 3 & $7,50 \%$ \\
\hline de & I & $2 \%$ & 2 & $5 \%$ & 3 & $5,90 \%$ & 2 & $3,90 \%$ \\
\hline con & 0 & - & I & & 2 & $3,90 \%$ & I & $2,50 \%$ \\
\hline \multicolumn{9}{|c|}{ Total } \\
\hline Correctas & & \multicolumn{2}{|c|}{$47,50 \%$} & \multicolumn{2}{|c|}{$49 \%$} & \multicolumn{2}{|c|}{$42,85 \%$} \\
\hline $\begin{array}{l}\text { Inc./en } \\
\text { blanco }\end{array}$ & \multicolumn{2}{|c|}{$76 \%$} & \multicolumn{2}{|c|}{$52,50 \%$} & \multicolumn{2}{|c|}{$51 \%$} & \multicolumn{2}{|c|}{$57,15 \%$} \\
\hline
\end{tabular}

\section{Discusión}

Una de las primeras utilidades que tiene para el docente la aplicación del $A E$ a un contenido problemático, como las preposiciones locativas, es la de contar con una estadística real que ayude a discernir qué unidades provocan un mayor número de errores y por qué. Asimismo, es un modo de realizar una autoevaluación, puesto que partiendo de estos datos se puede entrar en un ejercicio de reflexión sobre la efectividad de los métodos que se usan y que también influirán en la planificación temporal de la enseñanza.

En nuestro caso hemos constatado que, evidentemente, existen unidades a las que hay que prestar más atención que a otras. En general, gracias al gran 
hincapié que se hace en el aula a la falta de paralelismo en algunos modos de uso (el consabido ejemplo del uso de la preposición en italiano para referirnos a extensiones geográficas distintas) entre las preposiciones en ambas lenguas, el alumno es capaz de priorizar esta información y de buscar la solución correcta ante estos problemas. No en vano, la $\mathrm{Ol}$ y la $\mathrm{O} 2-\mathrm{AB}$ son las que registran menor número de errores.

El número de errores se incrementa para las oraciones $\mathrm{O} 3$ y $\mathrm{O} 4$, puesto que los usos de «da» y «per» como preposiciones locativas resultan más heterogéneos para el aprendiz; por ello, busca recursos próximos en su lengua frente al desconocimiento de la solución correcta. Ante este tipo de unidades, el docente tiene que llamar la atención para que el alumno sea capaz de prestar atención a los usos más complejos por motivos de tipo intralingüístico, es decir por el propio funcionamiento de la lengua (Greco, 2006:6).

El corpus recopilado podría constituir un primer paso hacia la creación de herramientas interactivas y ejercicios que tomen como referencia los casos donde haya más incidencia de errores y fomenten asimismo la repetición. Los métodos interactivos se han sumado a los métodos tradicionales de enseñanza de lenguas (como los instrumentales o los audio-orales) y han adquirido gran relevancia en los últimos años (Marangon, 2012). Existen herramientas online con las que el docente puede crear, sin tener apenas conocimientos informáticos, ejercicios interactivos de todo tipo. Posiblemente, el rasgo más idiosincrásico de este método es el aumento de la autonomía del alumno, lo cual ha multiplicado su importancia dentro de su propio proceso de aprendizaje. En el caso que nos ocupa lo más interesante sería la creación de ejercicios para completar huecos que solo admitan como correcta una única respuesta, en la línea de lo sugerido por Caballero-Rubio y Hernández-Corral (1998), o ejercicios contrastivos con ejemplos en ambas lenguas.

Otra de las utilidades del corpus compilado podría ser la de apoyar la explicación teórica de las unidades que se deben tratar con ejemplos del corpus. Es de vital importancia, como afirma Solsona-Martínez (2007) explicar contrastivamente las diferencias entre los paradigmas preposicionales de ambas lenguas e insistir en los casos más conflictivos. En este sentido, el corpus podrá servir de apoyo para explicar los valores de las preposiciones que más dificultad impliquen para el alumno. Asimismo también puede ser interesante realizar una exposición y explicación del propio corpus como manera de familiarizar al alumno con las estrategias docentes: si se va a hacer que el alumno trabaje con el corpus de un modo más autónomo, sería conveniente también explicarle el proceso de elaboración del mismo y sus utilidades, además de por qué es beneficioso el trabajo con esta herramienta. 
Desde el punto de vista del discente la consideración de ciertos fenómenos como problemáticos podría suponer un punto de partida para la proposición de un modelo de autoaprendizaje mediante inducción o mediante ejercicios. Mediante la mera visualización del corpus, el alumno puede aprender, además de preposiciones y colocaciones correctas, frases hechas o léxico de todo tipo de manera indirecta. Evidentemente, también se trata de una actividad de repetición que puede ayudar a asimilar los contenidos que menor transparencia de significado tengan (SolsonaMartínez, 2007).

$\mathrm{Si}$, como hemos sugerido anteriormente, se preparan actividades interactivas tomando como base el corpus (principalmente para evaluar a los alumnos sobre las unidades que más repaso necesitan), el aprendizaje se lleva a cabo de un modo más ameno y autónomo y se desarrolla la capacidad de autocorrección del discente (Caballero-Rubio y Hernández-Corral, 1998). Del mismo modo, el alumno también aprenderá qué no es correcto. La disposición de ejemplos reales, además, fomenta el interés del propio actante, al ser consciente de que lo que está visualizando son errores o fallos que son susceptibles de ser cometidos por él mismo en un futuro.

\section{Conclusiones}

Más allá de la compilación de grandes corpus para analizar fenómenos a nivel macrotextual, resulta interesante la compilación de corpus a pequeña escala en el aula de LE, especialmente en el caso de dos lenguas afines como son el español y el italiano. Una vez identificados y observados los problemas del alumno, y clasificado el fenómeno como problemático, el docente puede comenzar a planificar su enseñanza mediante la propuesta de distintas estrategias para solventar dichos problemas.

La incorporación de nuevos elementos a la didáctica de LE (sobre todo relacionados con las nuevas tecnologías) se ha convertido en una acción casi obligada en un paradigma de cambio en el que las clases magistrales con el profesor como único participante han dejado de ser atractivas y la figura del alumno ha adquirido importancia como actante en su propio proceso de aprendizaje.

Además, como se ha mencionado anteriormente, la incorporación de herramientas y métodos nuevos es particularmente necesaria en el caso de las lenguas afines, donde las dificultades se multiplican. Despertar el interés del alumno es una máxima fundamental para que logre incorporar a su acervo lingüístico los elementos que se le proponen diariamente en clase.

\section{Referencias bibliográficas}

CABALLERO-RUBIO, M. D. C., y HERNÁNDEZ-CORRAL, J. B. (1998). Las preposiciones locativas en español e italiano. In La enseñanza del español 
como lengua extranjera: Del pasado al futuro. Actas del VIII Congreso Internacional de ASELE (pP. 195-206).

CALVI, M. V. (2004). Aprendizaje de lenguas afines: español e italiano. Redele: Revista electrónica de Didáctica ELE, (I), 2.

CORDER, S. (1967). The significance of learners' errors. IRAL 5/4, 161-170, recogido en Error analysis and the interlanguage, 1981, 6-13.

CORPAS-PASTOR, G., Y SEGHIRI-DOMÍNGUEZ, M. (2006). El concepto de representatividad en la Lingüistica del Corpus: aproximaciones teóricas y metodológicas. Documento técnico BFF2003-046I6 MCYT/TI-DT-2006-I.

GRECO, S. (2006). Lenguas afines. Redele: Revista electrónica de Didáctica ELE, (6).

LENARDUZZI, R. (2003). Estrategias de aprendizaje y contrastividad: una propuesta de trabajo. EUT Edizioni Università di Trieste.

MARANGON, G. (20I2). Consideraciones en torno a los métodos de enseñanza de lenguas extrajeras y a las teorías de aprendizaje. Revista de Filología y Lingüistica de la Universidad de Costa Rica, | 2 | - 134.

PÉREZ-ÁVILA, E. A. (2006). El corpus lingüístico en la didáctica del léxico en el aula de E/LE. RedELE. Red Electrónica de Didáctica del Español como Lengua Extranjera, II, I-83.

PIÑOL, M. C. (2012). Lingüística de corpus y enseñanza del español como 2/L. Arco/Libros.

QUIÑONES, V. D. A. (2009). El análisis de errores en el campo del español como lengua extranjera: algunas cuestiones metodológicas. Revista Nebrija de Lingüistica aplicada a la enseñanza de Lenguas, (5).

SELINKER, L. (1969). Language transfer. General linguistics, 9(2), 67.

SELINKER, L. (1972). Interlanguage. IRAL-International Review of Applied Linguistics in Language Teaching, I0(I-4), 209-232.

SINCLAIR, J. (2004) Developing linguistic corpora: a guide to good practice en M. Wynne (ed.), Developing Linguistic Corpora: a Guide to Good Practice, pp. I20. University of Oxford: AHDS Literature, Languages and Linguistics.

SINCLAIR, J. (2004). Trust the text. London: Routledge.

SOLSONA-MARTÍNEZ, C. (2006). Los errores sistemáticos como evidencia de estadios de aprendizaje: asimilación de la preposición italiana da por parte de aprendices hispanohablantes de italiano/le. En Actas del XXXV Simposio Internacional de la Sociedad Española de Lingüistica (pp. 1770-1787).

SOLSONA-MARTÍNEZ, C. (2007). ¿Cómo se asimilan las preposiciones propias italianas? Acercamiento a la cuestión a partir del análisis del output de aprendices hispanohablantes de italiano/LE en la primera fase de su interlengua. Philologia Hispalensis, 2I(I).

SOLSONA-MARTÍNEZ, C. (2008). Estrategias empleadas por el aprendiz hispanohablante de italiano/L2. En 25 años de lingüistica en España hitos y retos (pp. 185-192). Universidad de Murcia. 
TOMASELLI, D. (2008). Italiano y español: dos lenguas diferentes. En Actas del VIII Congreso de Lingüistica General. Madrid: Laboratorio de Lingüística Informática.

VÁZQUEZ, G. (1992). Análisis de errores y aprendizaje de español/lengua extranjera, Frankfurt: Peter Lang.

VILLAVICENCIO-SIMÓN, Y. (2014). Enseñanza y aprendizaje del italiano para profesores de español como lengua extranjera en la Universidad de Oriente. Santiago, (134), 338-348. 